\title{
Seismic capacity of masonry groin vaults through upper bound limit analysis
}

\author{
A. Gaetani, G. Monti \& A. Paolone \\ Sapienza University of Rome, Italy \\ P.B. Lourenço \\ University of Minho, Guimarães, Portugal \\ G. Milani \\ Polytechnic of Milan, Italy
}

\begin{abstract}
A sensitivity analysis on the seismic capacity of masonry groin vaults, the simplest form of cross vault, is described in this paper. The main objectives of this study were: 1 ) assessing the effects and the influence of the main geometrical and mechanical properties, 2) proposing an analytical formulation for evaluating the seismic capacity of groin vaults (as a guidance to engineers and practitioners). In particular, the influence of the diameter, thickness, angle of embrace, infill and tensile strength, was investigated. On the other hand, the interaction with the rest of the structure was accounted only choosing two different boundary conditions. The analysis has been performed through a non-commercial software based on the upper bound approach of standard limit analysis. The results gave grounds for multiple linear regression analyses in order to get analytical equations for quick seismic evaluation of existing groin vaults.
\end{abstract}

\section{INTRODUCTION}

Clay brick, stone and masonry vaults are diffused all over the world with almost seven thousand years of history. Representing probably the first form of permanent dwelling in the prehistory, starting from the 12th century AD, masonry vaults reached in Europe such a level of beauty and technological perfection that still impresses the modern observer. However, although the relevance and the long-lasting history clearly prove an accurate design process for dead loads, the seismic vulnerability of these elements still represents an open and delicate issue in the conservation of historical buildings.

This aspect is emphasized by the systematic collection of damages occurred during strong Italian earthquakes of the last 40 years, displaying sometimes incalculable loss in terms of cultural heritage (Doglioni et al. 1994). The collapse of the cross vault frescoed by Giotto and Cimabue in the Basilica of St. Francis of Assisi in 1997 is an appalling example (Croci 2000). More recently, L'Aquila earthquake of 2009 damaged more than $70 \%$ of vaults of the inspected churches (Podestà et al. 2010).

In this regard, considering the cross vault as one of the most diffused and fascinating structural typologies of the European architectural heritage, the present paper deals with the seismic behavior of the groin vault, that is, the simplest kind of cross vaults obtained by the intersection at right angles of two semi-circular barrel vaults. The goal of this study was to evaluate the influence of the main geometrical parameters (diameter, thick-ness, angle of embrace), tensile strength, infill and boundary conditions on the seismic capacity of the vault. All the parameters are detailed in the following section.

Regarding the presence of the infill, in particular, several studies demonstrated its crucial role in assessing the capacity of masonry vaulted structures (Gilbert 2001; Cavicchi \& Gambarotta 2005; Cavicchi \& Gambarotta 2006; Milani \& Lourenço 2012). Croci (2000), for instance, demonstrated how the crisis of the cross vault in the Basilica of Assisi was due to the continuous accumulation of loose infill which, during the past earthquakes, facilitated increasing permanent deformations until the collapse occurrence.

In order to perform the sensitivity analysis, a noncommercial code implemented by one of the authors (Milani et al. 2009a; 2009b) was adopted. This is based on the kinematic theorem of the standard limit analysis (with associated flow rule) and for further details, the reader is referred to the cited papers.

With the aim of identifying the most frequent failure mechanisms, the results of the analysis have been visually inspected and ordered according to the input parameters. In reverse, this gave the possibility to heuristically deduce the range of parameters associated to a particular mechanism. Relating this catalogue to a multiple linear regression analysis provided valuable tools for quick seismic evaluation of groin vaults, which may represent the first step for filling the lack of recommendations in the current Codes of Practice. 


\section{ANALYSIS OVERVIEW}

\subsection{Boundary conditions}

Two boundary conditions were considered, namely "simply supported", "in-plane shear" (Figure 1). They are representative of two different scenarios within the buildings. The former regards the vaults with supports of the same stiffness, e.g. central nave columns. The latter deals with vaults in which the different stiffness of the supports allows a differential displacement, e.g. lateral nave with the colonnade more flexible than the lateral wall. It must be stressed that both conditions account only for the local behavior of the vault, neglecting any mutual interactions with the rest of the structure, even in case of in-plane shear where the pure sliding of one side does not consider the real stiffness of the supports.

Moreover, in order to properly simulate the equal horizontal displacement of the two sliding corners (e.g. two consecutive columns of a colonnade), an internal infinitely rigid constrain (strut/tie) has been implemented between the two rollers (upper side of Figure 1b). The aim of the fictitious constrain is

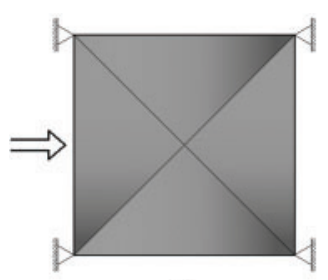

a)

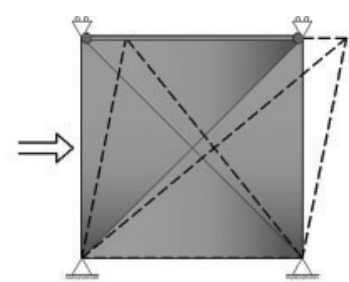

b)
Figure 1. Plan view and boundary conditions for groin vaults: a) simply supported; b) in-plane shear (strut/tie between rollers).

Table 1. Geometrical parameters of the sensitivity analysis.

\begin{tabular}{llll}
\hline Geometry & \multicolumn{3}{l}{ Adopted values } \\
\hline Diameter [m] & 3.6 & 4.5 & 5.4 \\
Thickness [diameter] & $1 / 20$ & $1 / 33$ & $1 / 50$ \\
Angle of embrace & $120^{\circ}$ & $130^{\circ}$ & $140^{\circ}$ \\
\hline
\end{tabular}

to avoid the unreal corner spread out due to the gravitational load thrust.

\subsection{Geometry}

Since the great importance geometry plays in the capacity of masonry structures, all the descriptive parameters have been considered, namely angle of embrace diameter, and thickness. The adopted values are reported in Table 1, where the thickness is accounted as a ratio over the diameter.

Regarding the angle of embrace, in order to provide consistent interface properties at the supports, the voussoirs underneath the last element of the vault were considered fully constrained (Figure 2a). Finally, in order to deal with in situ measurements, in the following, diameter and angle of embrace will be substituted by span and rise (Figure $2 b$ ).

\subsection{Infill as assigned load and mass}

For the sake of simplicity, the infill has been modelled as a distributed load and mass on the extrados of the vault, thus neglecting the proper distribution of vertical and horizontal pressure, the influence of the possible tensile strength (resulting as a slightly cemented loose material), and the nonlinear behavior of the infill during motion (active and passive pressure).

Although this approach is still poorly understood, Clemente (1997) suggested four different schematizations. Assuming the seismic action toward the right hand side, it is possible to consider: $I 1$ ) only the contribution of the horizontal stripes of the left hand part of the infill; I2) as I 1 but on both sides; I3) the contribution of vertical stripes of the infill on both sides; I4) an overall distributed horizontal load whose resultant is equal to the entire mass of the infill.

Figure 3 shows the four levels of infill adopted in the analysis, indicated by the central angle $0^{\circ}, 40^{\circ}, 60^{\circ}$, $90^{\circ}$, where $0^{\circ}$ conventionally stands for no infill while $90^{\circ}$ represents the case in which the vault is completely covered. Also in this case, with the aim of considering only in situ measurements, in the following, the infill will be addressed according to the vertical height at the corner which depends on the angle of embrace (Figure 2b).

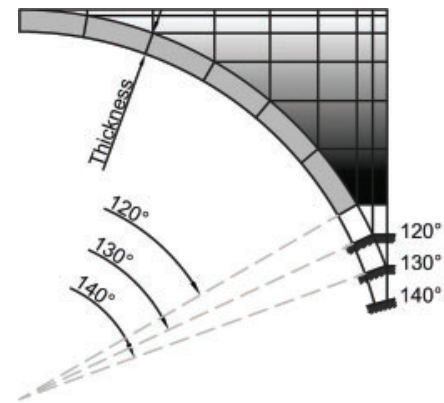

a)

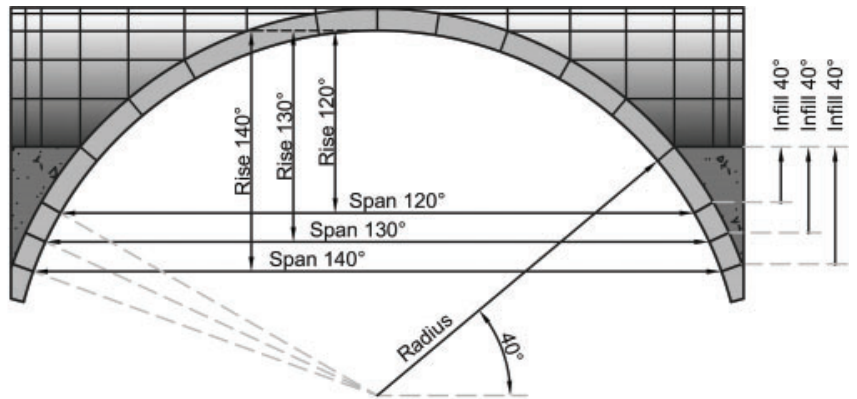

b)

Figure 2. Groin vault description: a) constrain location; b) span, rise and infill calculation. 


\subsection{Description of the model}

A total of 2106 analyses were performed. As two different boundary conditions are involved, namely simply supported and in-plane shear $(1053+1053$ analyses $)$, the results are split and presented in the following two sections. Moreover, for the sake of clarity, according to Figure 2, the parameters are defined as:

- $S$ span. In order to catch any possible scale effect, it is the unique geometrical parameter considered as a dimensional quantity $[\mathrm{m}]$.

$-R$ rise over span ratio

- Th thickness over span ratio

- I height of the infill over span ratio

- Ft tensile strength [MPa]

Regarding the infill, the categories $0,40,60,90^{\circ}$ will be preferred when a more concise description is requested.

As far as the mechanical parameters are concerned, they were mostly chosen on the basis of the averaged values recommended by the Italian code for good stone masonry and brick masonry with lime mortar $(\mathrm{CM}$ 2009) (Table 2). Great attention has been paid to the tensile strength since it is considered the most influent features (Lourenço 2002). In particular, three values were adopted, namely $\mathrm{Ft}=0.05,0.10,0.20 \mathrm{MPa}$, of which the minimum value may be accounted for the usual assumption of null strength.

Regarding the implemented code, it is based on the upper bound theorem of the limit analysis, that is, the solution is found through the minimization of the virtual powers. The bare vault was modelled by way of six noded rigid-infinitely resistant wedge elements with

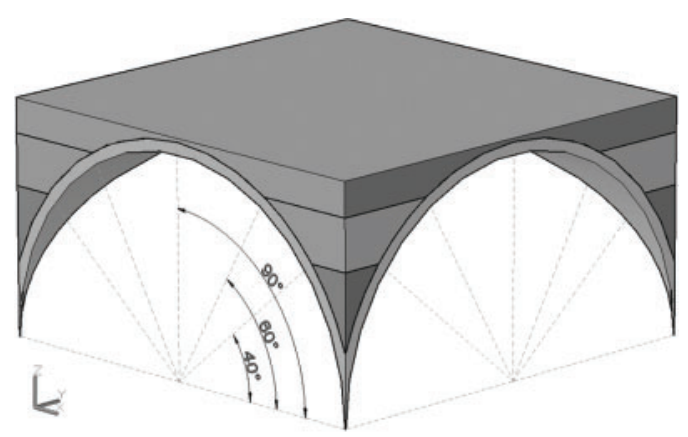

Figure 3. Infill schematization according to the central angle.

Table 2. Mechanical parameters of the sensitivity analysis.

\begin{tabular}{lll}
\hline Mass density sound masonry & $\rho_{\mathrm{s}}$ & $1.8 \mathrm{ton} / \mathrm{m}^{3}$ \\
Mass density loose masonry (infill) & $\rho_{l}$ & $1.2 \mathrm{ton} / \mathrm{m}^{3}$ \\
Compression strength & $\mathrm{Fc}$ & $3.2 \mathrm{MPa}$ \\
Tensile strength & $\mathrm{Ft}$ & $0.05,0.10$, \\
& & $0.20 \mathrm{MPa}$ \\
Compression cutoff & $\mathrm{Fc}_{\mathrm{cut}}$ & $6 \mathrm{Ft}$ \\
Cohesion & $\mathrm{c}$ & $1.5 \mathrm{Ft}$ \\
Friction angle & $\Phi$ & $30^{\circ}$ \\
Compression linearized cap angle & $\Phi_{2}$ & $60^{\circ}$ \\
\hline
\end{tabular}

velocities discontinuities allowed only along the edges of adjoining elements. Considering the associated flow rule, it is possible to calculate the velocity jumps for each interface, thus the power dissipated. The consequent problem is linear in terms of centroid velocities and load multiplier, allowing the application of linear programming technique.

The vault was discretized according to the directrix and generatrix of the webs. However, since the importance of the interface as a possible fracture line, the consequent net was refined in order to accommodate more general fracture mechanisms. As it is clearly comprehensible, the resulting mesh depicted in Figure 4 is not representative of any real pattern and the block interlocking is neglected. Moreover, in order to accommodate dry-jointed elements, their sides are concentric.

\section{SIMPLY SUPPORTED VAULT}

Considering the four different approaches proposed to model the infill, the first study was aimed at evaluating which one provided the lowest load multipliers in the largest number of cases. Therefore, neglecting the cases with null infill and null capacity (for which a comparison cannot be made), the study involved 230 analyses (4 infill schematizations each, thus 920 cases). Accordingly, $I 2$ resulted the most conservative schematization, leading also to the largest number of null capacity cases. As a consequence, in the subsequent discussion, only the results following the schematization $I 2$ will be considered.

\subsection{Failure mechanisms}

Considering only I2, 324 analyses (243 with and 81 without infill) were selected. In the fashion of the kinematic approach of the limit analysis, the study was aimed at identifying all the possible failure mechanisms. According to the wide range of input parameters adopted in the sensitivity analysis, these mechanisms can be regarded as the most plausible and representative ones for simply supported groin vaults.

However, since the proposed simple schematization is based on the visual inspection (by the authors), it is not expected to be complete or rigorous (sometimes

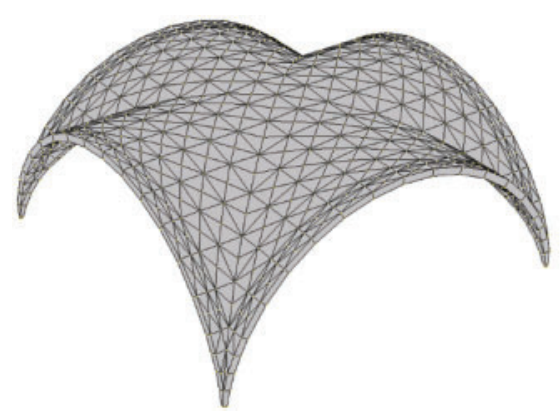

Figure 4. Mesh of the groin vault $(T h=1 / 50)$. 
different cases showed common features). Moreover, as in the case of macro-elements (Doglioni et al. 1994), the mechanisms were well-defined only with a good quality masonry, which means, in the present study, larger values of $\mathrm{Ft}$.

The results are collected in Table 3 together with the occurrence frequency. Discarding the three mechanisms with the lowest values (less than $1 \%$ ), the main ones are depicted in Figure 5. Since the symmetry of the problem, the mechanisms are basically in-plane, thus with strong similarity with the seismic behavior of masonry arches. However, in case of groin vaults, the presence of the webs forces the inner hinges to locate in the central part of the vault (where the vault is more flat, thus less stiff), largely within $\pm 20^{\circ}$ from the crown line.

\subsection{Range of input parameters}

Looking at the combination of the five parameters involved in the analysis, with the aim of define a possible range of values in which a single mechanism develops, all the data have been arranged in the form of box-plots reported in Figure 6. According to each parameter and each mechanism, the figure shows the first, second (median) and third quartile, together with maximum and minimum values, and possible outliers (circles). In reverse, for any given set of values that describes a real vault, it might be possible to evaluate the most plausible mechanism (or more than one in case of interval overlapping).

As can be seen, the results for infill equal to $0^{\circ}$ and $40^{\circ}$ are almost perfectly the same, which means that even small amount of debris at the vault corners do not affect the type of collapse failure. More in deep, considering the rise, only $4 \mathrm{H}$ and $\mathrm{R} \& 2 \mathrm{H}$ are associated to $R=0.29$ (flatter vault). On the other hand, if

Table 3. Occurrence frequency for simply supported groin vault.

\begin{tabular}{lll}
\hline Mechanisms & Abbr. & Frequency \\
\hline Four hinges & $4 \mathrm{H}$ & $49 \%$ \\
Two hinges and roller & $2 \mathrm{H} \& \mathrm{R}$ & $20 \%$ \\
Roller and two hinges & R\&2H & $17 \%$ \\
Two rollers & $2 \mathrm{R}$ & $6 \%$ \\
Null capacity & Null & $5 \%$ \\
Three hinges and clamp & - & $<1 \%$ \\
Clamp and three hinges & - & $<1 \%$ \\
Roller, hinge and clamp & - & $<1 \%$ \\
\hline
\end{tabular}

$R=0.35$ (the highest vaults of the database), $\mathrm{R} \& 2 \mathrm{H}$ never occurs. $R=0.35$ is also the unique value which led to vaults with null capacity.

Regarding the thickness, $T h=0.02$ led to only two mechanisms (and vaults with null capacity), namely $4 \mathrm{H}$ and $2 \mathrm{H} \& \mathrm{R}$, with a strong prevalence of the former. Furthermore, with the highest values of $T h$, almost all the vaults have a capacity larger than zero and a significant occurrence of $2 \mathrm{R}$ is notable. Finally, regarding the tensile strength, the lowest value $(0.05 \mathrm{MPa})$ did not lead to $\mathrm{R} \& 2 \mathrm{H}$ but, as expected, it was the only value with null capacity vaults.

In general, the capacity of the vault decreases as the span, the infill and the rise increase. On the other hand, the capacity increases as the tensile strength increases. Regarding the thickness, it contributes positively in case of $4 \mathrm{H}$ and $2 \mathrm{H} \& \mathrm{R}$, whereas negatively in case $\mathrm{R} \& 2 \mathrm{H}$ and $2 \mathrm{R}$. However, since the clear trend associated to rise and tensile strength, according to the database considered, they can be addressed as the most crucial parameters in determining the capacity of simply supported groin vaults.

\subsection{Multiple Linear Regression (MLR)}

MLR is a very well-known technique which allows finding (linear) relations between dependent and independent variables, that is, between the load multiplier $\lambda$ and the input parameters. The general prediction formula is reported in Equation 1. In the following, the estimated values are indicated with an overline, e.g. the value following from the limit analysis is labelled as $\lambda$ whereas the one from the regression model as $\bar{\lambda}$ ):

$$
\begin{aligned}
& \bar{\lambda}_{k}=\bar{\beta}_{0}+\sum_{i=1}^{p} \bar{\beta}_{i} x_{k i} \quad k=1 \ldots n \\
& \bar{\lambda}=\boldsymbol{X} \overline{\boldsymbol{\beta}} \\
& \bar{\lambda}=\left[\begin{array}{c}
\bar{\lambda}_{1} \\
\bar{\lambda}_{2} \\
\vdots \\
\bar{\lambda}_{n}
\end{array}\right] \overline{\boldsymbol{\beta}}=\left[\begin{array}{c}
\bar{\beta}_{0} \\
\bar{\beta}_{1} \\
\vdots \\
\bar{\beta}_{p}
\end{array}\right] \quad \boldsymbol{X}=\left[\begin{array}{cccc}
1 & x_{11} & \ldots & x_{1 p} \\
1 & x_{21} & \ldots & x_{2 p} \\
\vdots & \vdots & & \vdots \\
1 & x_{n 1} & \ldots & x_{n p}
\end{array}\right]
\end{aligned}
$$

where $\bar{\lambda}$ is the vector of the $k$ observations, $\overline{\boldsymbol{\beta}}$ the vector of the regression coefficients ( $\bar{\beta}_{0}$ is the intercept), $\boldsymbol{X}$ is the design matrix with $p$ predictors, namely $S, R, T h, I$ and Ft. It must be stressed that there is no need to discuss about multicollinearity because each parameter has its own physical meaning. This approach, although
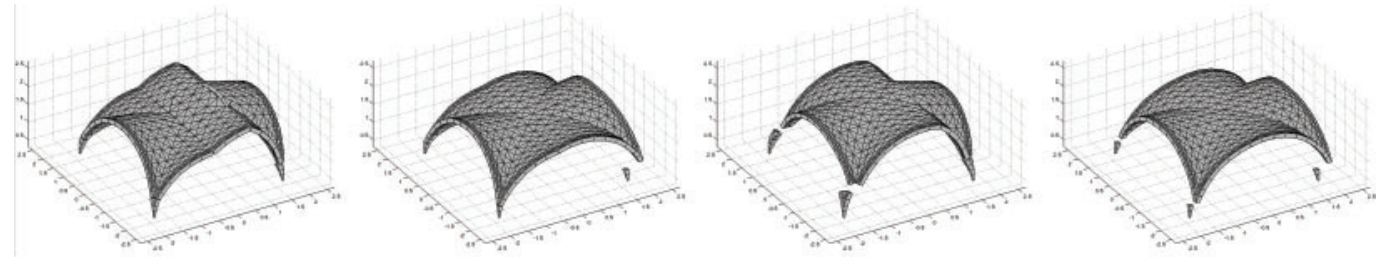

Figure 5. Most frequent mechanisms for simply supported groin vaults (4H, 2H\&R, R\&2H, 2R). 
lacking of a deep physical support, it is still considered appropriate for having valuable information (even only mathematical) to predict the horizontal load multiplier and to assess the influence of each parameter. Finally, in order to get rid of less significant parameters, a procedure named Stepwise Regression was adopted, identifying the smallest set of predictors with a significance close to the maximum.

In order to determine the unknown regression coefficients of $\overline{\boldsymbol{\beta}}$ in Equation 1, the Ordinary Least Square method is applied, which is based on the minimization of the sum of squared residuals. The values of the regression coefficients are collected in Equation 2 where $S$ and $F t$ are in [m] and [MPa], respectively, and all the other parameters are dimensionless.

According to the previous considerations, the results of MLR are shown in form of scatter diagrams in Figure 7, where the limit analysis outcomes are reported in abscissa and the predicted values in ordinate, i.e. underestimated values below the bisector. As it is possible to see, the simple relationships proposed for determining $\bar{\lambda}$ are in good agreement with the limit analysis results. In the diagrams, the coefficient of determination $\mathrm{R}^{2}$ is also reported for each model, being considerably large except for the $2 \mathrm{R}$ model for which the poor database did not allow a more accurate prediction.

$$
\begin{aligned}
& \bar{\lambda}_{4 H}=2.58-0.17 S-5.91 R+14.24 T h-1.34 I+5.86 F t \\
& \bar{\lambda}_{2 H \& R}=3.70-0.13 S-9.38 R+6.77 T h-0.51 I+3.34 F t \\
& \bar{\lambda}_{R \& 2 H}=7.08-0.24 S-17.07 R-1.21 I+5.41 F t \\
& \bar{\lambda}_{2 R}=1.42-0.14 S-0.61 I
\end{aligned}
$$

\section{Tensile strength [MPa]}
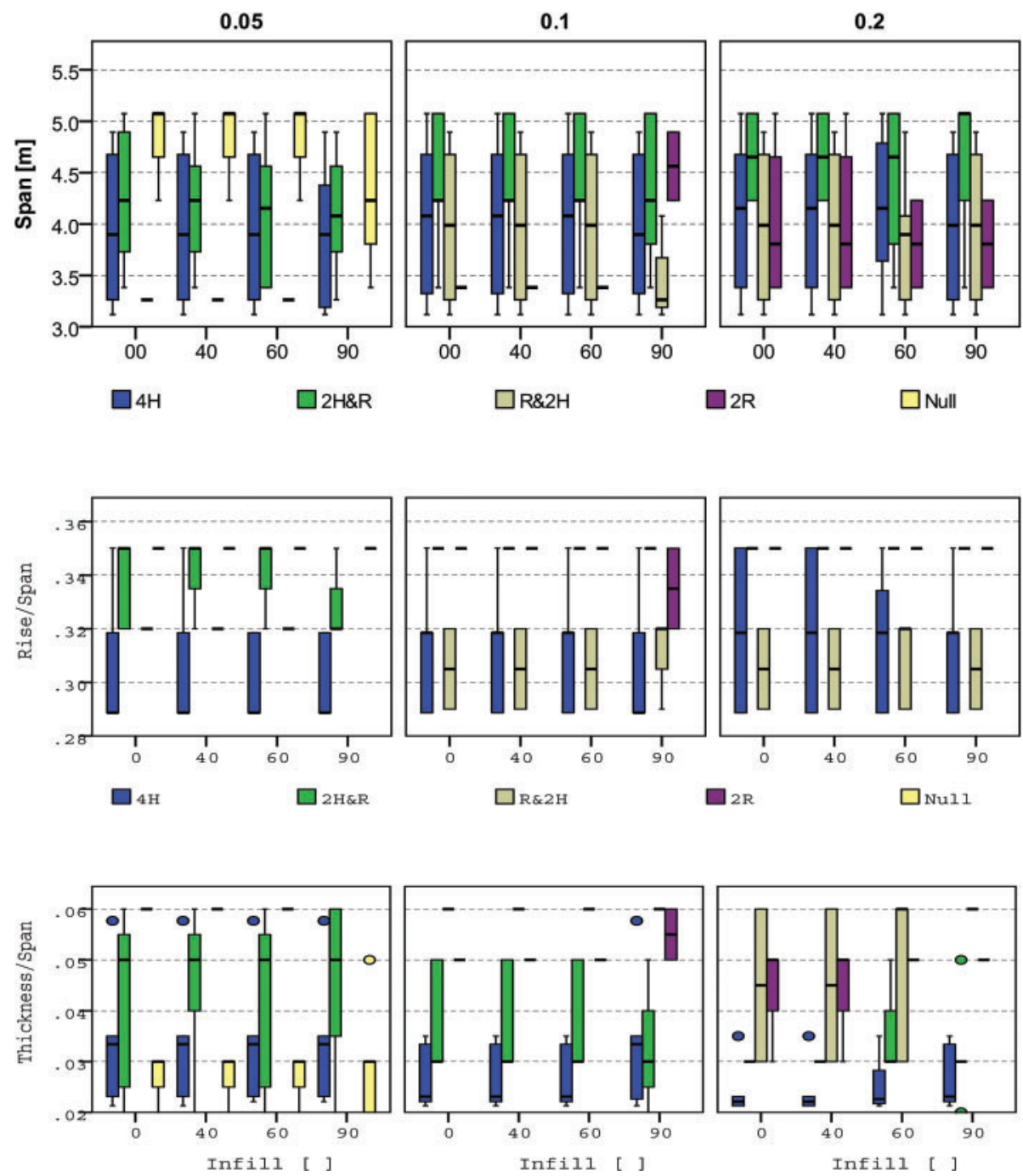

Figure 6. Ranges of the input parameters for each failure mechanism (simply supported). 

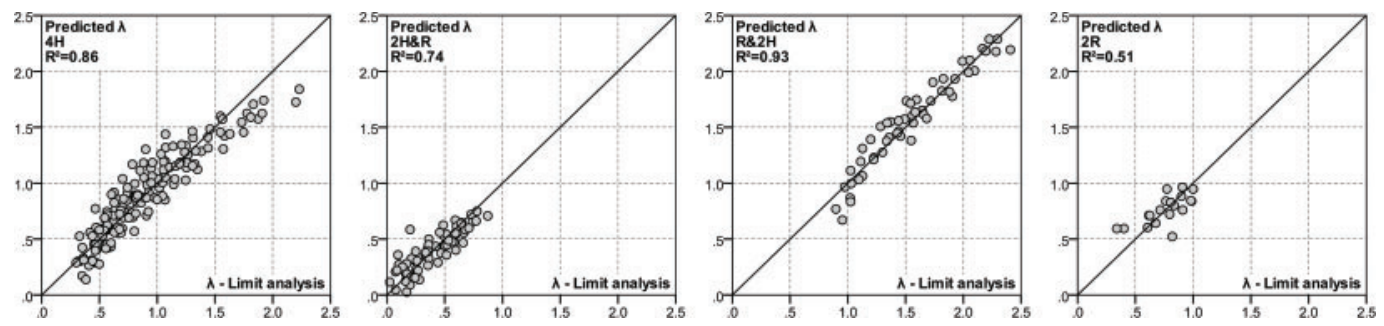

Figure 7. Scatter plots of the prediction models according to MLR (simply supported).

Table 4. Occurrence frequency groin vaults subjected to in-plane shear.

\begin{tabular}{lll}
\hline Mechanisms & Abbr. & Frequency \\
\hline Bending 1 & B1 & $30 \%$ \\
Sliding 1 & S1 & $12 \%$ \\
Sliding 2 & S2 & $10 \%$ \\
Diagonal & D & $10 \%$ \\
Bending 2 & B2 & $9 \%$ \\
Bending 3 & B3 & $8 \%$ \\
Null & Null & $7 \%$ \\
Sliding 3 & S3 & $3 \%$ \\
Others & - & $<10 \%$ \\
\hline
\end{tabular}

\section{IN-PLANE SHEAR}

This section basically parallels the previous one and the reader is referred to it for further explanations.

In particular, the infill schematization $I 2$ resulted again the most conservative one, leading also to the largest number of null capacity cases. Consequently, in the subsequent discussion, only the results following the schematization $I 2$ will be considered.

\subsection{Failure mechanism}

Considering only $I 2,324$ analyses (243 with and 81 without infill) were selected. In the fashion of the kinematic approach of the limit analysis, the study was aimed at identifying all the possible failure mechanisms, which can be regarded as the most plausible and representative ones for the groin vault subjected to in-plane shear.

The results are collected in Table 4 together with the occurrence frequency. Since the three-dimensional behavior of the vault, a larger amount of mechanisms was detected and only the ones that covered $90 \%$ of all the cases will be considered in the following. Three bending (B) and three sliding (S) mechanisms were detected, together with the one labelled as "diagonal" (D). All of them are depicted in Figure 8.

\subsection{Range of input parameters}

Since the difficulties posed by the combination of the five parameters in achieving a straightforward range of values in which a single mechanism develops, all the data have been arranged in the form of box-plots reported in Figure 9. Accordingly, for any given set of parameters that describe a real vault, it is possible to evaluate the most plausible mechanism (or more than one in case of interval overlapping).

Also in this case, the range of parameters for infill equal to $0^{\circ}$ and $40^{\circ}$ are almost perfectly the same, which means that even small amount of debris at the vault corners do not affect the type of collapse failure.

More in general, since the notably difficulty arisen with three-dimensional mechanisms, it is not easy to detect clear trend in the mechanism occurrence. The increment of the span did not produce significant changes, unless for B2 occurrence, which decreases, and B3 and Null's which increase. Moreover, incrementing the level of the infill (from bare to completely covered vault), S1, S2 and B2 occurrence decreases whereas the occurrence of null capacity vaults increases.

Looking at the material properties, Null and B3 are present only in case of low $F t$, whereas $\mathrm{S} 1, \mathrm{~B} 2$ and $\mathrm{S} 3$ are present only with higher values.

Finally, as far as the load multiplier $(\lambda)$ is concerned, the capacity of the vault decreases as the infill and the rise increase. On the other hand, it is possible to catch an inverse relationship with the tensile strength. Regarding the other parameters, there are no appreciable trends. However, in general terms, according to actual database, the groin vaults with $R=0.35$ and $F t=0.05 \mathrm{MPa}$ led to a horizontal load multiplier lower than 0.8 , whereas the largest values can be reached only with $\mathrm{S} 1$ and $\mathrm{B} 2$ mechanisms.

\subsection{Multiple linear regression}

The results of MLR are reported in form of scatter diagrams in Figure 10, whereas the values of the regression coefficients are collected in Equation 3, where $S$ and $F t$ are measured in [m] and [MPa], respectively, and all the other parameters are dimensionless.

$$
\begin{aligned}
& \bar{\lambda}_{B 1}=1.06-0.92 S-2.23 R+12.72 T h-0.82 I+2.93 F t \\
& \bar{\lambda}_{S 1}=5.83-1.79 S-13.95 R-3.68 T h-0.70 I+2.34 F t \\
& \bar{\lambda}_{S 2}=1.54-0.73 S-6.27 R+17.24 T h-0.32 I+4.42 F t \\
& \bar{\lambda}_{D}=0.36-0.81 S-0.23 I+2.40 T h+1.63 F t \\
& \bar{\lambda}_{B 2}=3.23-1.80 S-8.30 R+7.96 T h-1.03 I+4.46 F t \\
& \bar{\lambda}_{B 3}=1.31-0.69 S-3.07 R+4.28 T h-0.36 I+3.86 F t \\
& \bar{\lambda}_{S 3}=0.32+13.80 T h-0.32 I
\end{aligned}
$$



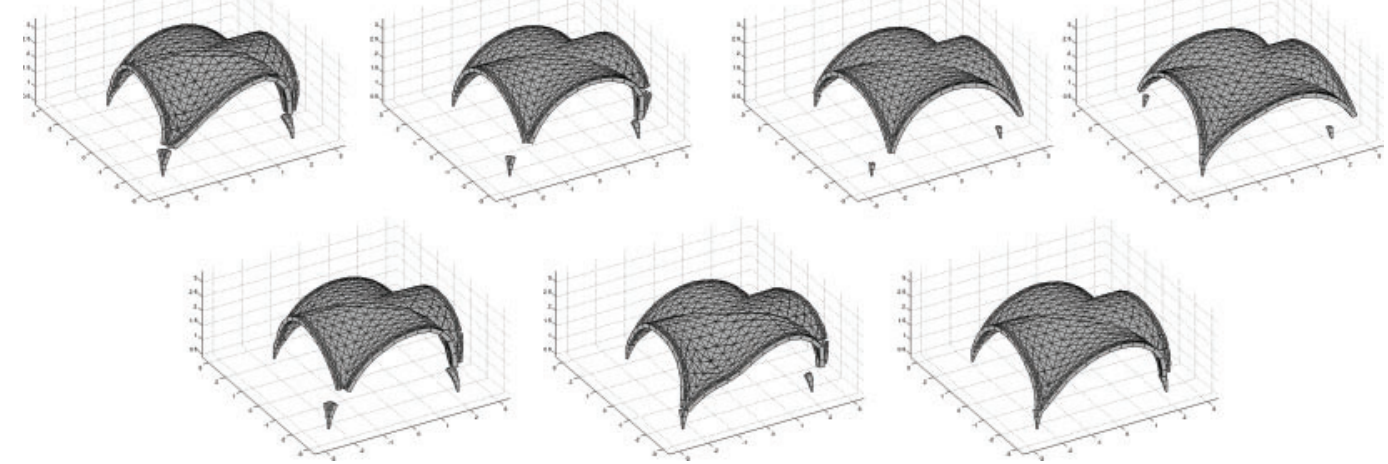

Figure 8. Most frequent mechanisms for groin vaults subjected to in-plane shear (B1, S1, S2, D, B2, B3, S3).

Tensile strength [MPa]
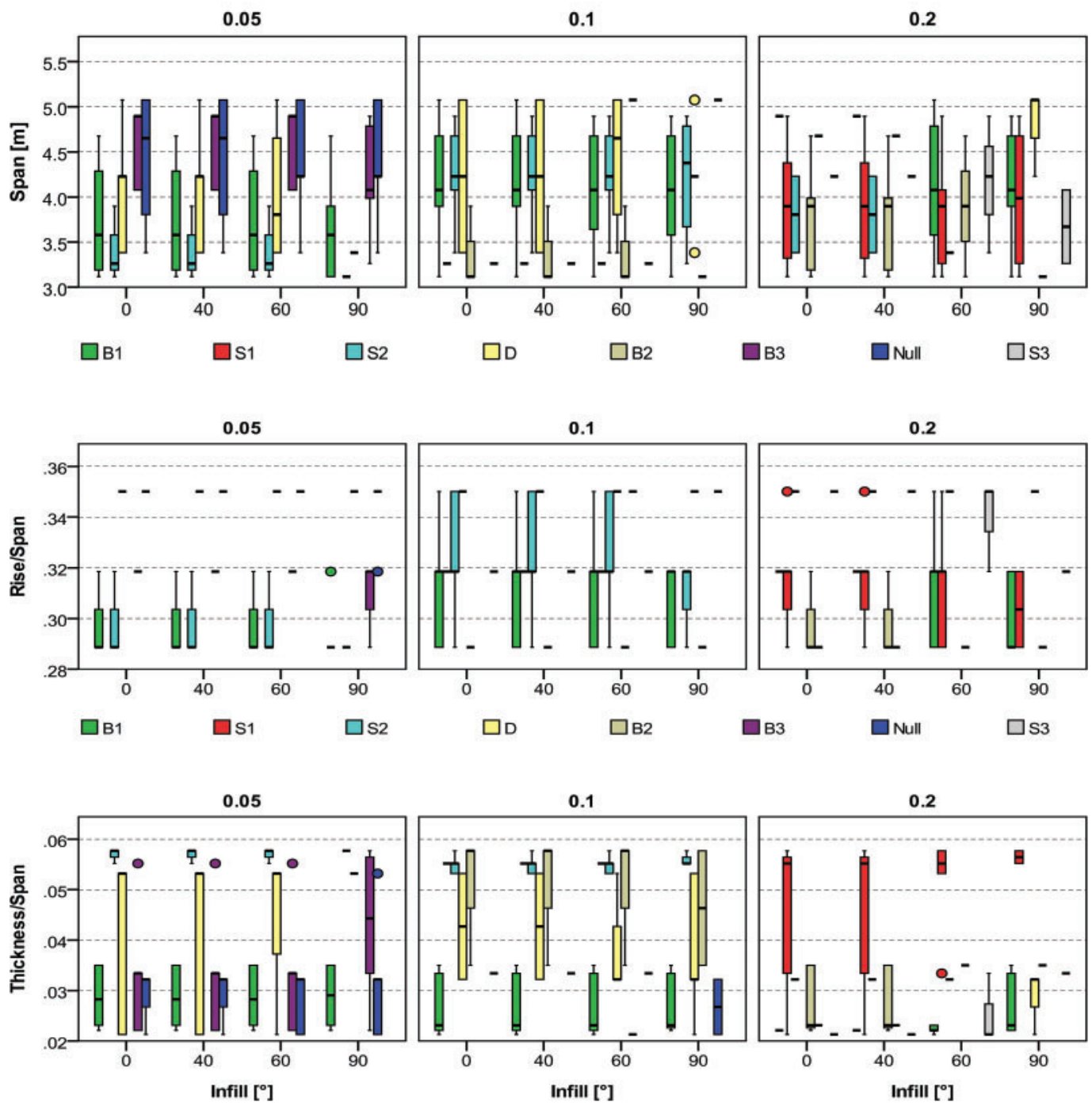

Figure 9. Ranges of the input parameters for each failure mechanism (in-plane shear). 

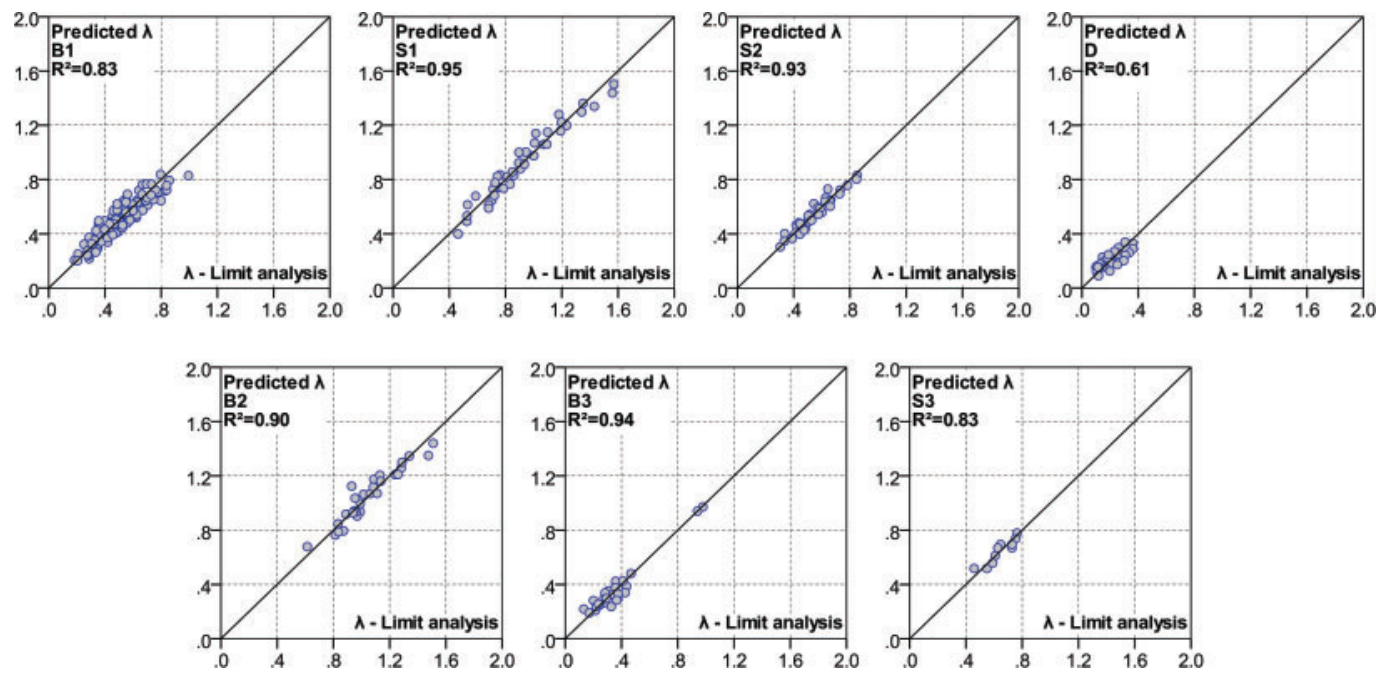

Figure 10. Scatter plots of the prediction models according to MLR (in-plane shear).

As it is possible to see in Figure 10, the simple relationships proposed for determining $\bar{\lambda}$ are in good agreement with the limit analysis results. In the diagrams the coefficient of determination $\mathrm{R}^{2}$ is reported for each model, being considerably large except for the D mechanism (with $\bar{\lambda}$ lower than 0.4).

\section{CONCLUSIONS}

This paper has presented the results of a sensitivity analysis on the seismic capacity of masonry groin vaults. The objective was to investigate the influence of the geometrical parameters, as well as the tensile strength, the boundary conditions, and the presence of the infill. The main outcomes of this study can be summarized as follows.

Although approximated, among the four infill schematizations examined, considering the contribution of horizontal stripes on both sides of the vault provided the lowest load multipliers in the largest number of cases.

Regarding the boundary conditions, the simply supported vault showed, as expected, a behavior similar to the masonry arch one. However, the presence of the perpendicular webs forced the internal hinges to locate close to crown where the stiffness is lower. On the other hand, the vault subjected to in-plane shear showed a more complicated behavior and more effort is still requested on this topic. Looking at the professional field, a schematization of the vault by way of arch of variable thickness and equivalent arch assemblage, for the two cases respectively, is rather desirable.

Finally, the Multiple Linear Regression analysis (based on the inspection of the failure mechanisms) provided valuable results that can support the analyst in assessing the seismic capacity of groin vaults. Future steps may interest their validation with experimental tests and more sophisticated analyses.

\section{REFERENCES}

Cavicchi, A. \& Gambarotta, L., 2005. Collapse analysis of masonry bridges taking into account arch-fill interaction. Engineering Structures, 27(4): 605-615.

Cavicchi, A. \& Gambarotta, L., 2006. Two-dimensional finite element upper bound limit analysis of masonry bridges. Computers \& Structures, 84(31-32): 2316-2328.

Clemente, P., 1997. La verifica degli archi a conci lapidei, ENEA, Unità comunicazione e informazione.

CM, 2009. Instruction for the application of the Building Standard for Constructions. Circular 2 February 2009, n. 617 [Italy]

Croci, G., 2000. General methodology for the structural restoration of historic buildings: the cases of the Tower of Pisa and the Basilica of Assisi. Journal of Cultural Heritage, 1(1): 7-18.

Doglioni, F. et al., 1994. Le chiese e il terremoto. Dalla vulnerabilità constatata nel terremoto del Friuli al miglioramento antisismico nel restauro. Verso una politica di prevenzione, Trieste: Lint Editoriale Associati.

Gilbert, M., 2001. RING: A 2D rigid block analysis program for masonry arch bridges. In 3rd International Arch Bridges Conference. Paris, 109-118.

Lourenço, P.B., 2002. Computations on historic masonry structures. Progress in Structural Engineering and Materials, 4(3): 301-319.

Milani, G. \& Lourenço, P.B., 2012. 3D non-linear behavior of masonry arch bridges. Computers \& Structures, 110-111: 133-150.

Milani, G., Milani, E. \& Tralli, A., 2009a. Upper Bound limit analysis model for FRP-reinforced masonry curved structures. Part I: Unreinforced masonry failure surfaces. Computers \& Structures, 87(23-24): 1516-1533.

Milani, G., Milani, E. \& Tralli, A., 2009b. Upper bound limit analysis model for FRP-reinforced masonry curved structures. Part II: Structural analyses. Computers \& Structures, 87(23-24): 1534-1558.

Podestà, S. et al., 2010. Damage assessment and seismic vulnerability of churches: the Abruzzo earthquake. Ingegneria Sismica, XXVII(1): 21-35. 